ARTICLE

DOI: $10.1038 / s 41467-018-06009-3$

\title{
Long-lived polarization memory in the electronic states of lead-halide perovskites from local structural dynamics
}

Jasmine P.H. Rivett ${ }^{1}$, Liang Z. Tan (10 2 ${ }^{2}$, Michael B. Price ${ }^{1}$, Sean A. Bourelle ${ }^{1}$, Nathaniel J.L.K. Davis ${ }^{3}$, James Xiao', Yatao Zou ${ }^{4}$, Rox Middleton (D) ${ }^{5}$, Baoquan Sun ${ }^{4}$, Andrew M. Rappe (i) ${ }^{2}$, Dan Credgington (D) ${ }^{1}$ \& Felix Deschler (i) ${ }^{1}$

Anharmonic crystal lattice dynamics have been observed in lead halide perovskites on picosecond timescales. Here, we report that the soft nature of the perovskite crystal lattice gives rise to dynamic fluctuations in the electronic properties of excited states. We use linear polarization selective transient absorption spectroscopy to study the charge carrier relaxation dynamics in lead-halide perovskite films and nanocrystals. We find that photo-excited charge carriers maintain an initial polarization anisotropy for several picoseconds, independent of crystallite size and composition, and well beyond the reported timescales of carrier scattering. First-principles calculations find intrinsic anisotropies in the transition dipole moment, which depend on the orientation of light polarization and the polar distortion of the local crystal lattice. Lattice dynamics are imprinted in the optical transitions and anisotropies arise on the time-scales of structural motion. The strong coupling between electronic states and structural dynamics requires a unique interpretation of recombination and transport mechanisms.

\footnotetext{
${ }^{1}$ Cavendish Laboratory, University of Cambridge, JJ Thomson Avenue, CB3 OHE Cambridge, UK. ${ }^{2}$ Department of Chemistry, University of Pennsylvania, Philadelphia, PA 19104, USA. ${ }^{3}$ School of Chemical and Physical Sciences, Victoria University of Wellington, Wellington 6140, New Zealand. ${ }^{4}$ Institute of Functional Nano \& Soft Materials (FUNSOM), Soochow University, 199 Ren'ai Road, 215123 Suzhou, People's Republic of China. ${ }^{5}$ Department of Chemistry, University of Cambridge, Lensfield Road, CB2 1EW Cambridge, UK. Correspondence and requests for materials should be addressed to

D.C. (email: djnc3@cam.ac.uk) or to F.D. (email: fd297@cam.ac.uk)
} 
$\mathrm{M}$ etal-halide perovskites of composition $\mathrm{ABX}_{3}$ (where $\mathrm{A}$ $=\mathrm{CH}_{3} \mathrm{NH}_{3}$ or $\mathrm{Cs}, \mathrm{B}=\mathrm{Pb}$, and $\mathrm{X}=\mathrm{I}, \mathrm{Br}$, or $\mathrm{Cl}$ ) are a recent class of solution-processable crystalline semiconductors. Perovskite solar cell power conversion efficiencies now exceed $20 \%$, and efficient light emitting devices with high color purity have been reported ${ }^{1}$. Their excellent photovoltaic performance derives from the perovskites' band-like semiconducting behavior, with high absorption coefficients ${ }^{2}$ in the range of $10^{5} \mathrm{~cm}^{-1}$, combined with long carrier lifetimes ${ }^{3,4}$. These materials lie between the extremes of highly-ordered, crystalline semiconductors, which can exhibit ballistic charge transport, and disordered, molecular semiconductors, where strong electron-phonon coupling leads to highly localized excited states. Lattice distortions, such as lattice phonons, and structural dynamics are key to understand the physics of these materials ${ }^{5,6}$. Recent reports have studied lead halide perovskite lattice dynamics on ultrafast timescales using diffraction ${ }^{7,8}$, Raman $^{9}$, and transient optical Kerr-effect ${ }^{10,11}$ experiments. Further, the combination of strong spin-orbit coupling and local electric fields generated in a non-centrosymmetric crystal lattice ${ }^{12-14}$ can give rise to Rashba-type symmetry breaking in carrier momentum space $^{15}$. Signatures of the Rashba effect have been detected in lead halide perovskites at low temperatures, however the range of reported splitting energies is large $(4-240 \mathrm{meV})^{16,17}$.

Here, we use linear polarization selective transient absorption spectroscopy (LP-TA) to investigate how the reported crystal dynamics affect the electronic states occupied by photoexcited carriers in lead halide perovskite thin films with organic and inorganic A-site cations $\left(\mathrm{CH}_{3} \mathrm{NH}_{3} \mathrm{PbX}_{3}, \mathrm{X}=\mathrm{I}, \mathrm{Br}, \mathrm{CsPbBr} \mathrm{Pb}_{3}\right)$ and nanocrystals $\left(\mathrm{CsPbI}_{3}\right)$ at room temperature. This method is sensitive to the coupling between the optical polarization vector of the absorbed light and the transition dipole matrix (TDM) element of the electronic states, which allows us to probe optical anisotropies in the excited state population. Optical alignment upon linearly-polarized excitation occurs in a range of semiconductors, with a variety of underlying causes. In GaAs, the dependence of the optical TDM on the angular momentum of the electronic wave functions imprints a short-lived anisotropic carrier momentum distribution on the excited state population ${ }^{18-21}$. This is lost through femtosecond carrier-carrier scattering. By contrast, optical alignment in molecular materials, with more localized excitonic states, may arise from an alignment of the TDM with physical structure $22-25$. Loss of polarization memory in this case arises from physical reorientation of the photoexcited molecule or diffusion of the excited state to regions with different dipole matrix orientation. Here, we show that lead halide perovskites lie between these two extremes. Their soft structural nature allows dynamic symmetry breaking of the delocalized electronic states, preserving optical alignment far beyond the timescale of momentum-scattering events. Optical alignment is lost on the timescales of local structural reorientation rather than diffusion.

\section{Results}

Linear polarization selective transient absorption. Thin film lead halide perovskite samples were prepared using standard procedures ${ }^{26}$. $\mathrm{CH}_{3} \mathrm{NH}_{3} \mathrm{PbI}_{3}$ films were deposited on fused silica in nitrogen atmosphere by spin-coating from a 3:1 molar solution of $\mathrm{CH}_{3} \mathrm{NH}_{3} \mathrm{I}$ and either $\mathrm{PbCl}_{2}$ or $\mathrm{Pb}\left(\mathrm{CH}_{3} \mathrm{COO}\right)_{2}$. $\mathrm{CH}_{3} \mathrm{NH}_{3} \mathrm{PbI}_{3}$ bulk films are continuous (thickness of approximately $300 \mathrm{~nm}$ ) with a polycrystalline domain size of a few $\mu \mathrm{m}$ for the chloride precursor and a few $100 \mathrm{~nm}$ for the acetate precursor (Supplementary Figure 1). $\mathrm{CsPbI}_{3}$ nanocrystals were synthesized according to the procedure of Protesescu et al. ${ }^{27}$ with an average size of $14 \mathrm{~nm}$ and a cubic shape (Supplementary Figure 1), and thin films were prepared by spin-coating a $10 \mathrm{mg} \mathrm{ml}^{-1}$ nanocrystal solution onto fused silica at $2000 \mathrm{rpm} . \mathrm{CH}_{3} \mathrm{NH}_{3} \mathrm{PbBr}_{3}$ films were deposited on fused silica in nitrogen atmosphere by spin-coating from a 3:1 molar solution of $\mathrm{CH}_{3} \mathrm{NH}_{3} \mathrm{Br}$ and $\mathrm{Pb}$ $\left(\mathrm{CH}_{3} \mathrm{COO}\right)_{2}$. The optical properties of the thin films are consistent with previous literature reports, and we confirmed that the samples show no intrinsic optical anisotropy (Fig. 1a) using steady state linear absorption measurements. We estimate the optical band gap to be at $1.6 \mathrm{eV}\left(\mathrm{CH}_{3} \mathrm{NH}_{3} \mathrm{PbI}_{3}\right), 1.8 \mathrm{eV}\left(\mathrm{CsPbI}_{3}\right)$, and $2.3 \mathrm{eV}\left(\mathrm{CH}_{3} \mathrm{NH}_{3} \mathrm{PbBr}_{3}\right)$. CdSe/CdS nanocrystals were prepared using the method of Bae et al. ${ }^{28}$ with diameter of $12 \mathrm{~nm}^{29}$ and optical band gap of $1.8 \mathrm{eV}$.

Samples were excited with linearly polarized pump pulses (pulse length less than $50 \mathrm{fs}$, approximately $25 \mathrm{meV}$ FWHM) at energies between 10 and $200 \mathrm{meV}$ above the optical bandgap and probed after a variable time delay with linearly polarized probe pulses with broad spectral range $(1.55-2.55 \mathrm{eV})$. We selectively recorded the polarization component of the probe pulses aligned parallel or perpendicular to the linear polarization of the pump pulses directly after the sample. This arrangement avoids artifacts from polarization-dependent reflection or excitation intensity changes (Fig. 1b). Probe transmission spectra through the sample with $\left(T_{\text {on }}\right)$ and without $\left(T_{\text {off }}\right)$ the pump pulse are used to calculate the relative change in transmission following photoexcitation $\frac{\Delta T}{T}=\frac{T_{\text {on }}-T_{\text {off }}}{T_{\text {off }}}$.
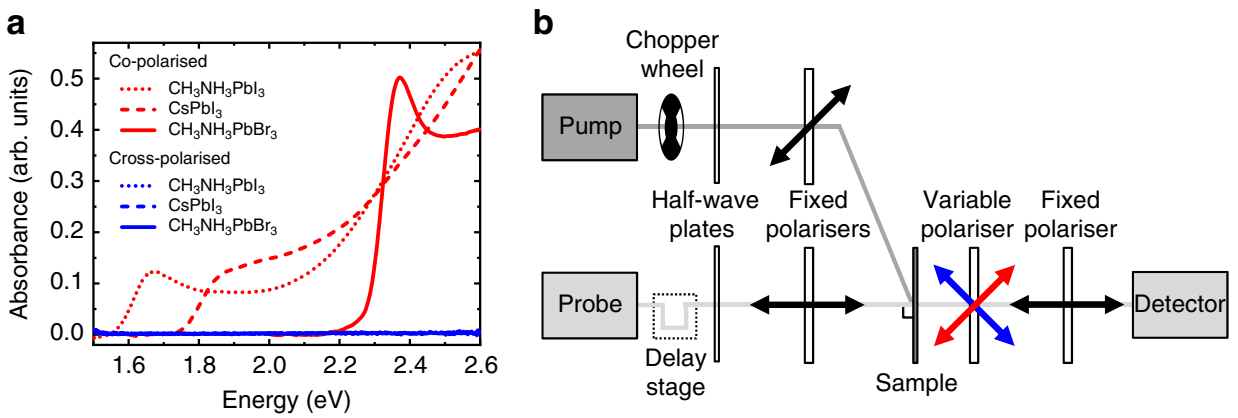

Fig. 1 Sample properties and experimental setup. a Steady state linear absorption spectra of the investigated hybrid perovskite thin films. In co-polarized measurements (red), polarisers placed before and after the sample are aligned parallel. In cross-polarized measurements (blue), polarisers placed before and after the sample are aligned perpendicular. No intrinsic dichroism is observed. $\mathbf{b}$ Sketch of the experimental setup used for polarization selective transient absorption spectroscopy. The linear polarization of the pump pulse is set at an angle of $45^{\circ}$ with respect to the linear polarization of the probe pulses before the sample. A second set of polarizers after the sample detects the linear polarization component of the probe pulses either parallel or perpendicular to the pump pulse polarization direction 

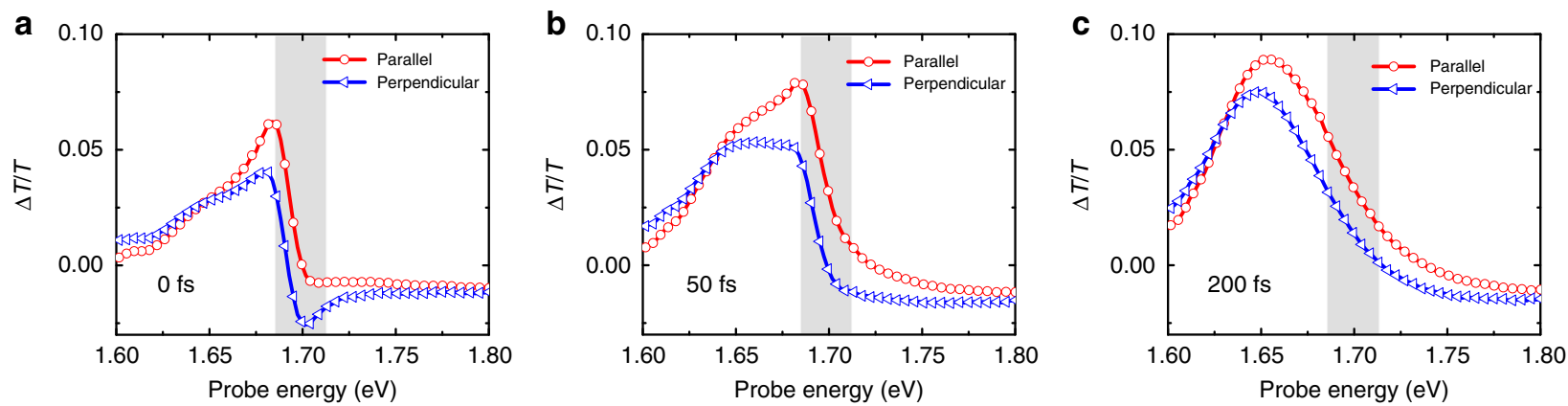

Fig. 2 Ultrafast polarization selective transient absorption. a-c Spectrally resolved sub-picosecond transient absorption response of $\mathrm{CH}_{3} \mathrm{NH}_{3} \mathrm{Pbl}_{3}$ hybrid perovskite thin films at the indicated time delays. The sample was excited with laser pulses of energy $1.7 \mathrm{eV}$ (FWHM approximately 25 meV, shaded area), pulse length of approximately $50 \mathrm{fs}$, and excitation fluence approximately $20 \mu \mathrm{Jm}^{-2}$. The change in the transmission of the probe was measured at the indicated time delays with the linear polarization of the probe pulse aligned either parallel (red) or perpendicular (blue) to the linear polarization of the pump pulse. We observe a stronger increase in transmission (photoinduced bleach signal) when the pump and probe pulse polarizations are aligned parallel. The differences observed in the transient spectral dynamics indicate that the carrier populations probed in parallel and perpendicular configurations undergo different relaxation
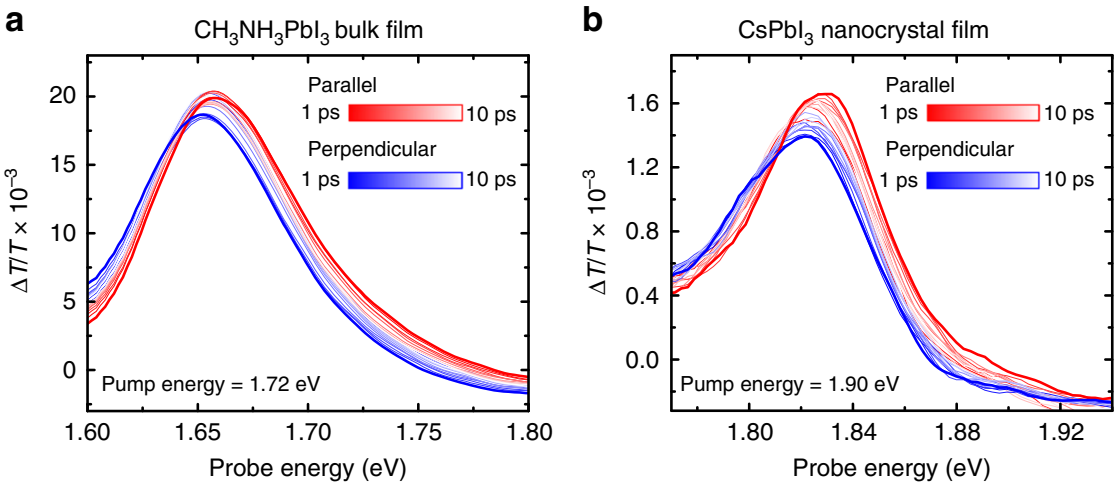

Fig. 3 Spectrally resolved picosecond transient absorption spectra. a $\mathrm{CH}_{3} \mathrm{NH}_{3} \mathrm{Pbl}_{3}$ hybrid perovskite bulk film (excitation approximately $1.72 \mathrm{eV}, 15 \mu \mathrm{J} \mathrm{cm}{ }^{-2}$ ) and $\mathbf{b} \mathrm{CsPb}_{3}$ nanocrystal film (excitation approximately $1.90 \mathrm{eV}, 4 \mu \mathrm{J} \mathrm{cm}{ }^{-2}$ ). The samples were excited with laser pulses of FWHM approximately 25 meV and pulse length of approximately $50 \mathrm{fs}$. The change in transmission of the probe was measured at the indicated time delays with the linear polarization of the probe pulse aligned parallel (red) or perpendicular (blue) to the linear polarization of the pump pulse. The initially polarization-dependent spectra converge within the first $10 \mathrm{ps}$ after photoexcitation

Control samples of CdSe/CdS nanocrystals excited at $1.91 \mathrm{eV}$ and probed between 1.78 and $1.91 \mathrm{eV}$ show no detectable anisotropy in $\Delta T / T$ within our time-resolution, as expected (Supplementary Figure 2). Figure 2 presents the spectrally resolved values for $\Delta T / T$ for $\mathrm{CH}_{3} \mathrm{NH}_{3} \mathrm{PbI}_{3}$ films excited at $1.7 \mathrm{eV}$.

Immediately after photoexcitation $(t=0 \mathrm{fs}$, Fig. $2 \mathrm{a})$ we observe a strong positive signal close to the excitation energy in both polarization configurations, representing a bleach of the groundstate transitions by a population of excited carriers. The weak tail of this bleach extending towards the band edge at $1.64 \mathrm{eV}$ indicates that a small fraction of carriers has started to thermalize, while a large fraction of carriers remains non-thermal, i.e., these have not yet scattered with other carriers and phonons. The signal in the parallel configuration is stronger than in the perpendicular configuration, indicating that the parallel probe interacts with either a larger carrier population, or the excited carrier population exhibits a different TDM for different linear polarization directions. We take this as a first indication that the symmetry in the optical properties is broken by the linear polarization of the pump pulse. At later time delays ( $t=50 \mathrm{fs}$, Fig. $2 \mathrm{~b}$ ), the signal in the perpendicular configuration is strongly broadened towards the band edge, which we take as a sign that these carriers have thermalized further and started to cool. Unexpectedly, the signal in parallel configuration remains rather localized at energies close to the excitation energy (gray area). This suggests that the carrier population probed in parallel configuration has relaxed less. The differences in signal intensity remain, and neither distributions have yet fully reached a thermalized distribution. By $200 \mathrm{fs}$ (Fig. 2c), both parallel and perpendicular spectra evolve to spectral shapes well-described by thermalized Fermi-Dirac distributions and are now dominated by a strong positive signal close to the band edge, which has previously been assigned to phase-space filling by photoexcited carriers ${ }^{5,30}$. We note that the signal probed in perpendicular configuration maintains a different spectral shape and exhibits lower signal intensity by approximately $30 \%$. These persistent differences in signal intensity and shape suggest that the initial anisotropy is preserved through thermalization and cooling processes, such as carrier-carrier and carrier-lattice scattering.

We now discuss the anisotropy decay dynamics in the LP-TA of $\mathrm{CH}_{3} \mathrm{NH}_{3} \mathrm{PbI}_{3}$ over picosecond timescales in Fig. 3a. Two differences are apparent: The overall bleach intensity is higher for the parallel configuration, and the spectral shapes are different. Specifically, the bleach signal in the parallel configuration is higher on the high-energy side of the spectrum, while the opposite behavior is found on the low-energy side. The differences in spectral shape and intensity reduce over time, both converging to an intermediate spectrum with equal intensities by 

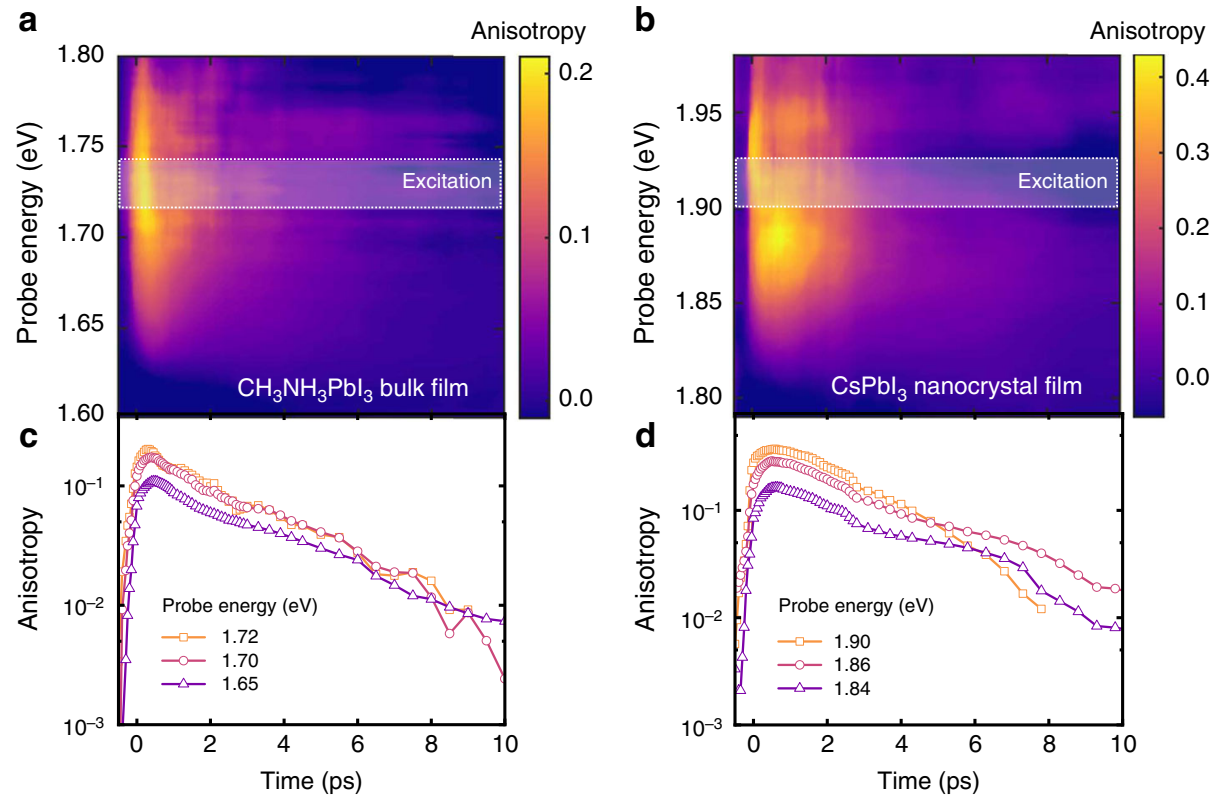

Fig. 4 Spectrally and temporally resolved polarization anisotropy maps. a $\mathrm{CH}_{3} \mathrm{NH}_{3} \mathrm{Pbl}_{3}$ hybrid perovskite bulk film (excitation at approximately $1.72 \mathrm{eV}, 2 \mu \mathrm{J}$ $\mathrm{cm}^{-2}, 50 \mathrm{fs}$ ) and $\mathbf{b} \mathrm{CsPbl}_{3}$ nanocrystal film (excitation at approximately $1.90 \mathrm{eV}, 3 \mu \mathrm{J} \mathrm{cm}-2,50 \mathrm{fs}$ ). A constant photoinduced absorption background was offset before calculating the polarization anisotropy. During the first $10 \mathrm{ps}$ after photoexcitation, the polarization anisotropy shifts to states closer to the band minima. Polarization anisotropy decay kinetics of $\mathbf{c} \mathrm{CH}_{3} \mathrm{NH}_{3} \mathrm{Pbl}_{3}$ hybrid perovskite bulk film and $\mathbf{d} \mathrm{CsPb}_{3}$ nanocrystal film. After initial relaxation, the anisotropy signal decays exponentially with a time constant of $2.85 \pm 0.1 \mathrm{ps}$ in the $\mathrm{CH}_{3} \mathrm{NH}_{3} \mathrm{Pbl}_{3}$ bulk film and $2.45 \pm 0.2 \mathrm{ps}$ in the $\mathrm{CsPbl}$ nanocrystal film. No significant variation in this decay time is found for different probe energies

approximately $10 \mathrm{ps}$. We extract LP-TA kinetics at the center of the photoinduced bleach signal $\left(1.64 \mathrm{eV}\right.$ in the $\mathrm{CH}_{3} \mathrm{NH}_{3} \mathrm{PbI}_{3}$ bulk film) to study the impact on population decay on the observed anisotropy. A linear superposition of parallel and perpendicular kinetics, equivalent to a magic angle pump-probe measurement, estimates the total population kinetics (Supplementary Figure 3). This value remains stable to longer than $10 \mathrm{ps}$, as expected at the excitation densities used, which indicates that negligible recombination occurs on the timescale over which the anisotropy is lost.

We observe similar anisotropy dynamics in $\mathrm{CH}_{3} \mathrm{NH}_{3} \mathrm{PbI}_{3}$ bulk films formed from both acetate and chloride precursors, and in $\mathrm{CsPbI}_{3}$ nanocrystal films (Fig. 3b, Supplementary Figure 3 and 4). At the excitation densities used, we only form one excitation on average per nanocrystal, which excludes interactions between multiple excited carriers as the origin of the anisotropy decay. Similar anisotropy effects are also observed for $\mathrm{CH}_{3} \mathrm{NH}_{3} \mathrm{PbBr}_{3}$ bulk films ${ }^{31}$ and $\mathrm{CsPbBr}_{3}$ bulk films (Supplementary Figures 57 ). We conclude that the polarization memory has a fundamental origin beyond the macroscopic crystal structure and is not determined by diffusion between crystallites ${ }^{32}$.

Polarization anisotropy maps and dynamics. Polarization anisotropy maps give insight into spectral dynamics of the anisotropy. In accordance with previous literature, we use a standard definition of polarization anisotropy ${ }^{33} A$ as a function of time and probe energy from the difference between LP-TA signals obtained in parallel $\left(\frac{\Delta T}{T}\right)_{\|}$or perpendicular $\left(\frac{\Delta T}{T}\right)_{\perp}$ configurations:

$$
A=\frac{\left(\frac{\Delta T}{T}\right)_{\|}-\left(\frac{\Delta T}{T}\right)_{\perp}}{\left(\frac{\Delta T}{T}\right)_{\|}+2 \cdot\left(\frac{\Delta T}{T}\right)_{\perp}}
$$

Immediately after photoexcitation, the anisotropy is largest near the pump energy in both $\mathrm{CH}_{3} \mathrm{NH}_{3} \mathrm{PbI}_{3}$ thin films (Fig. 4a) and $\mathrm{CsPbI}_{3}$ nanocrystal films (Fig. 4b). Within the first picosecond after excitation, the anisotropy maximum relaxes towards lower energies, also visible by an increase in the anisotropy rise times (up to $150 \mathrm{fs}$ ) towards the band edge (Supplementary Figure 8). The following decay of the anisotropy after thermalization can be quantified from the anisotropy kinetics at selected probe energies (Fig. 4c, d). The signal follows a single-exponential decay with a lifetime of $2.85 \pm 0.1 \mathrm{ps}$ for the $\mathrm{CH}_{3} \mathrm{NH}_{3} \mathrm{PbI}_{3}$ bulk film and $2.45 \pm 0.2 \mathrm{ps}$ for the $\mathrm{CsPbI}_{3}$ nanocrystal film, respectively (Supplementary Figure 9). No significant variation in these decay times is found for other probe energies, which suggests that the loss of the photoexcited anisotropy occurs through a common process for all states. These timescales are much longer than typical phonon emission $^{34-36}$ and carrier cooling times ${ }^{5,30,37}$, but are in agreement with reported timescales for dynamic fluctuations of the crystal lattice 8,38 . We take these observations as an indication that the origin for the persistent polarization anisotropy relates to the crystal conformation, rather than a particular carrier-phonon interaction. The similarity of the timescales, independent of crystallite size, suggests that the observed anisotropy is not lost through grain boundary scattering or diffusion between crystallites $^{32}$, but through processes operating on considerably smaller length-scales.

Different pump energies create excited states at different positions in the band, which allows probing the dependence of the polarization anisotropy on the properties of the initially excited state (Fig. 5). The anisotropy is measured at a fixed energy near the peak of the photoinduced bleach $(1.71 \mathrm{eV}$ for $\mathrm{CH}_{3} \mathrm{NH}_{3} \mathrm{PbI}_{3}$ acetate films, $1.69 \mathrm{eV}$ for $\mathrm{CH}_{3} \mathrm{NH}_{3} \mathrm{PbI}_{3}$ chloride films, $1.87 \mathrm{eV}$ for $\mathrm{CsPbI}_{3}$ nanocrystal films, $2.37 \mathrm{eV}$ for $\mathrm{CH}_{3} \mathrm{NH}_{3} \mathrm{PbBr}_{3}$ films) and averaged over the first $10 \mathrm{ps}$ in the iodide samples and over the first 1 ps in the bromide sample. The pump energy was varied from 1.7 to $2.5 \mathrm{eV}$, while keeping the excitation density constant. We find that the anisotropy is largest for pump energies close to resonance with the probe energy, and it decreases as the difference between pump and probe energy increases. We fitted the pump energy dependence of the 
a

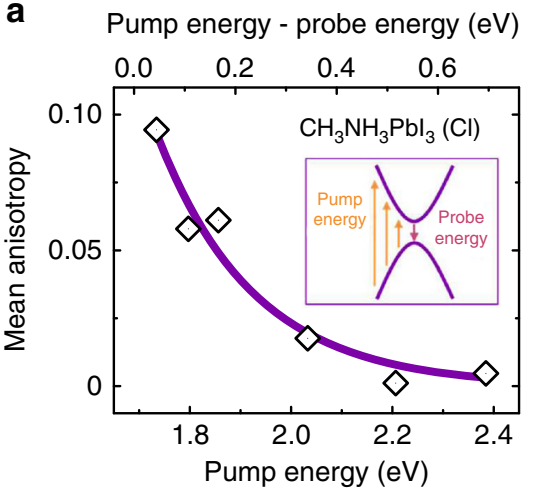

C

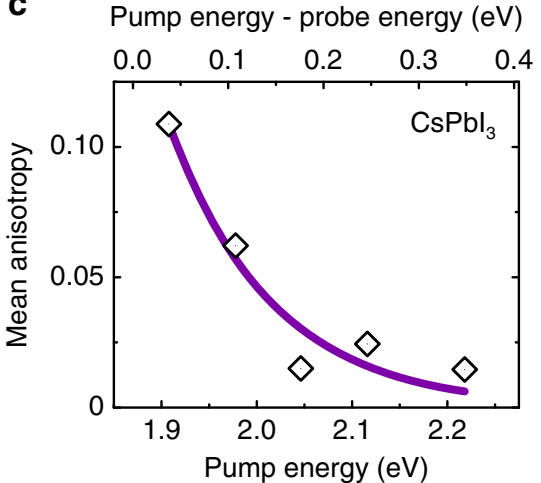

b

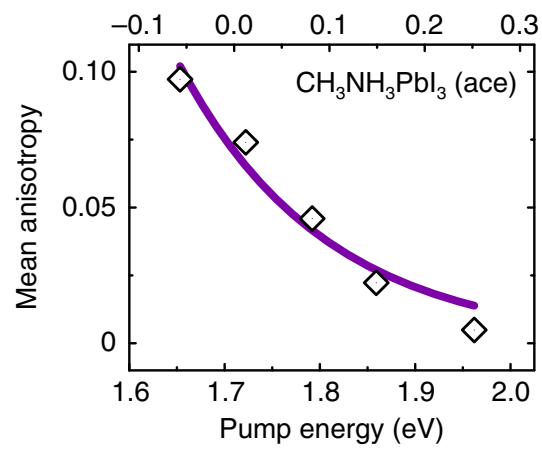

d

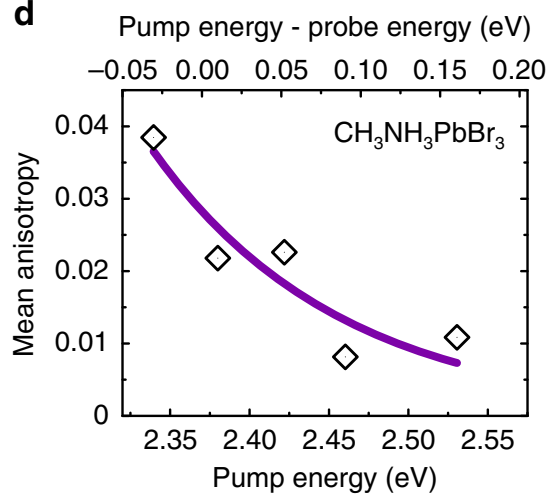

Fig. 5 Change in polarization anisotropy with pump energy. a $\mathrm{CH}_{3} \mathrm{NH}_{3} \mathrm{Pbl}_{3}$ bulk films prepared with chloride precursor, $\mathbf{b} \mathrm{CH}_{3} \mathrm{NH}_{3} \mathrm{Pbl}{ }_{3}$ bulk films prepared with acetate precursor, $\mathbf{c ~ C s P b l} 3$ nanocrystal films and $\mathbf{d ~} \mathrm{CH}_{3} \mathrm{NH}_{3} \mathrm{PbBr}_{3}$ bulk films. The energy per pulse was approximately $2 \mu \mathrm{J} \mathrm{cm}-2\left(\mathrm{CH}_{3} \mathrm{NH}_{3} \mathrm{Pbl}{ }_{3} \mathrm{Cl}\right), 16$ $\mu \mathrm{J} \mathrm{cm}^{-2}\left(\mathrm{CH}_{3} \mathrm{NH}_{3} \mathrm{Pbl}_{3}\right.$ acetate), $40 \mu \mathrm{J} \mathrm{cm}^{-2}\left(\mathrm{CsPb}_{3}\right)$, and $1 \mu \mathrm{J} \mathrm{cm}-2\left(\mathrm{CH}_{3} \mathrm{NH}_{3} \mathrm{PbBr}_{3}\right)$. The mean anisotropy was defined as the temporal and spectral average of the polarization anisotropy over the first $10 \mathrm{ps}$ (iodide samples) or first $1 \mathrm{ps}$ (bromide sample), over a $40 \mathrm{meV}$ probe window near the bleach maximum. The data is fitted with single exponentials, with decay constants of 190 meV $\left(\mathrm{CH}_{3} \mathrm{NH}_{3} \mathrm{Pbl}_{3} \mathrm{Cl}\right), 155 \mathrm{meV}\left(\mathrm{CH}_{3} \mathrm{NH}_{3} \mathrm{Pbl}_{3}\right.$ acetate), 110 meV $\left(\mathrm{CsPb}_{3}\right)$, and $120 \mathrm{meV}\left(\mathrm{CH}_{3} \mathrm{NH}_{3} \mathrm{PbBr}_{3}\right)$

anisotropy with an exponential decay. The characteristic energies are approximately $150 \mathrm{meV}$ (Fig. 5), which is over an order of magnitude higher than the dominant phonon modes of the material (approximately $50 \mathrm{~cm}^{-1}=6 \mathrm{meV}$ ). In measurements with either increasing pump energies (Supplementary Figure 10) or increasing excitation density (Supplementary Figure 11), we observe a reduction in the polarization anisotropy peak value, but not its lifetime.

To investigate the link between optically polarized excitation and probing, and the observed anisotropy, we turn to modeling and first principles calculations of the perovskite band structure (full details in Supplementary Methods 1 and 2). We consider a $\sqrt{2} \times \sqrt{2} \times 2$ unit cell of the tetragonal phase $\mathrm{CH}_{3} \mathrm{NH}_{3} \mathrm{PbI}_{3}$ structure. We use a structure with the MA molecules in a polar configuration which is known to be a local energy minimum for this unit cell ${ }^{39}$. This structure therefore serves as a local electronic structure model for $\mathrm{CH}_{3} \mathrm{NH}_{3} \mathrm{PbI}_{3}$. It has a polar axis in the $z$ direction, which breaks the local inversion symmetry. This causes spin-splitting of the conduction and valence bands, resulting in a Rashba-type band structure axially symmetric about the Rashba direction (here along the $z$-axis). Our calculations show that the breaking of the inversion symmetry has consequences not only for the band energies, but also for the transition dipole matrix elements. In Fig. 6a, b we plot the calculated TDM and ground state absorption close to the absorption edge. We find that the absorption is stronger when the light polarization axis is perpendicular to the Rashba direction, and weaker when it is parallel to the Rashba direction.
Given that no static polarization anisotropy is observable in our samples, we consider the local polarization of such nanoregions to be randomly oriented in space. We now discuss how excitation of such an isotropic distribution of polarized structures leads to overall polarization anisotropy. The theoretically calculated anisotropy in absorption is imprinted onto the carrier populations upon optical excitation. The total transient absorption signal for a sample containing many polarized nanoregions can be written in a condensed form as

$$
\alpha=\sum_{i \in \mathrm{NR}} \Pi_{i} \times n_{i},
$$

where $\mathrm{II}_{i}$ is transition dipole matrix element squared in nanoregion $i$, and $n_{i}$ is the density of available optical transitions in that nanoregion. Because each region has a different Rashba axis, $\mathrm{II}_{i}$ and $n_{i}$ depend on nanoregion and cannot be treated as constant. The larger the TDMs along the light polarization axis, the more carriers will be excited by the pump, and the lower $n_{i}$ will be. For the parallel and perpendicular configurations, the correlations between $\mathrm{II}_{i}$ and $n_{i}$ will be different in magnitude. This leads to different values of $\alpha$ and generates the polarization anisotropy, as quantified below.

To understand how TDM anisotropy relates to polarization anisotropy, we first expand II and $n$ as a series of spherical harmonics in Rashba direction and Brillouin zone k-vector. We then use these expansions to obtain expressions for transient absorption in parallel and perpendicular polarization 

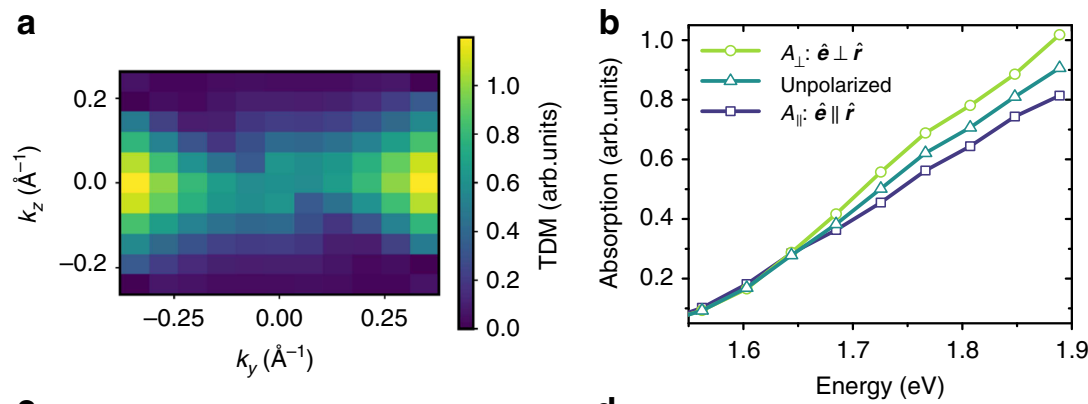

C

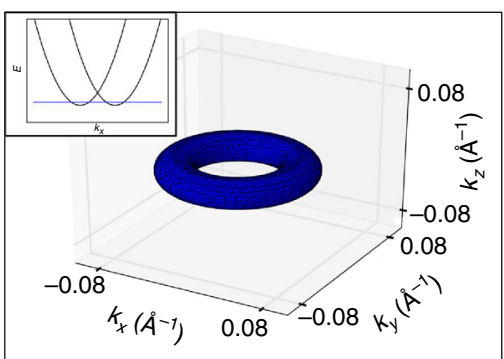

d

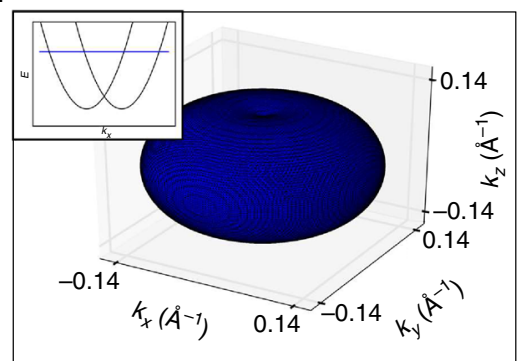

Fig. 6 First-principles calculation of transition dipole moments (TDMs). a Plot of the TDM $\mathbf{k}$-space asymmetry for energies close to the band minima. b Calculated absorption spectrum from first principles for electric field polarization direction ê parallel and perpendicular to the Rashba direction of the crystal lattice $\hat{r}$, and for unpolarized light. Iso-energetic surfaces of photoexcited carrier distributions for photon energies $\mathbf{c}$ above and $\mathbf{d}$ near the band edge. The insets show parabolic band disperisons and indictae the energies at which the iso-energetic $\mathbf{k}$-space surfaces were calculated

configurations. This is done by integrating contributions to transient absorption and integrating over Brillouin zone kvectors, and averaging over all possible Rashba directions, assumed isotropically distributed across nanoregions. Finally, we show that the polarization anisotropy can be written as

$$
A=-\frac{3}{2} \frac{C_{20}}{C_{00}} \frac{B_{20}}{B_{00}}
$$

where $C_{\mathrm{lm}}$ and $B_{\mathrm{lm}}$ are the coefficients of the expansions of $\mathrm{II}_{i}$ and $n_{i}$ in spherical harmonics. We estimate values of $C_{\operatorname{lm}}$ from our DFT calculations by fitting ab-initio TDMs to a spherical harmonic expansion. The $B_{\mathrm{lm}}$ are likewise obtained from DFT calculations because the carrier densities induced by the pump are determined by the TDMs. We obtain a value of $A=0.09$ for the polarization anisotropy of $\mathrm{CH}_{3} \mathrm{NH}_{3} \mathrm{PbI}_{3}$, for pump and probe energies of $1.7 \mathrm{eV}$. Comparing this theoretical estimate to the magnitudes of the maximum measured anisotropy immediately after excitation (Fig. 4) indicates that this mechanism is largely responsible for the observed polarization anisotropy. As this theoretical estimate is based on a single structure at a local energy minimum, it is likely that it will be increased if other nonequilibrium structures with higher polarity are considered as well. As the structure fluctuates on picosecond time scales, the Rashba axis changes stochastically in each nanoregion. We assume that this stochastic evolution approximates a random walk, with nanoregions losing memory of their initial Rashba directions after a characteristic time $\tau$. Over this time scale, the values of $\mathrm{II}_{i}$ become randomized and uncorrelated with $n_{i}$. The transient absorption signals for both parallel and perpendicular configurations would then regress towards the uncorrelated value $\langle\alpha\rangle=$ $\langle\Pi\rangle \times\langle n\rangle$ in the long-time limit, leading to a loss of polarization anisotropy, as in seen in Fig. 4.

Our theoretical model above assumes an isotropic distribution of carrier momenta shortly after photoexcitation-i.e., that thermalization and cooling processes randomize carrier momentum on approximately $200 \mathrm{fs}$ time scales ${ }^{40}$. This is likely to hold for excitations with more than approximately $100 \mathrm{meV}$ excess energy above the band edge, for which the excitation isosurface is approximately spherical (Fig. 6c, isosurface plot, high energy). However, excitations close to the band edge access a restricted momentum space, since the presence of polar distortions leads to toroidal isosurfaces (Fig. 6d, isosurface plot, low energy). To demonstrate how the changes in the momentum space distribution with energy affect transient absorption, we write the transient absorption signal for a single nanoregion $i$ as an integral over momentum space:

$$
\alpha_{i}=\int_{S} \mathrm{~d}^{3} k \Pi_{i}(\overrightarrow{\mathbf{k}}) n_{i}(\overrightarrow{\mathbf{k}})
$$

Here, the momentum resolved TDMs $\operatorname{II} i(\overrightarrow{\mathbf{k}})$ and the energy isosurface $S$ at the probe energy depend only on the probe energy and the Rashba direction. On the other hand, the momentum resolved carrier densities $n_{i}(\overrightarrow{\mathbf{k}})$ depend on the pump energy and the Rashba direction. As the pump energy is increased with respect to the band gap, the carrier density isosurface at excitation becomes progressively more spherical, and hence less correlated with the Rashba direction. This leads to decreasing correlation between $\alpha_{i}$ and Rashba direction with increasing separation between pump and probe energies. The dependence of polarization anisotropy on pump energy (Fig. 5) is consistent with this interpretation. However, we cannot rule out an additional influence from more energetic carriers losing excess energy to the lattice as they relax to the band edges, which could result in more rapid structural deformations of the lead-halide lattice and a partial randomization of the initial Rashba directions. Similarly, we cannot rule out a contribution from short-range diffusion of carriers between very small nanoregions, which may occur on similar timescales to structural deformation. However, the observed timescales and dependencies on pump and probe energy are difficult to reconcile with this process alone.

To model the energy dependence of transient absorption spectra, we insert the first-principles absorption spectra (Fig. 6b) 
into the analytical model for TA developed by Price et al. (Supplementary Method 3)..$^{5}$ This uses the Kramers-Kronig relations to consider the changes in both absorption and reflection caused by the excited carrier population, including state-filling via the Burstein-Moss effect and taking in to account Coulombic enhancement (Elliot's model of absorption) and band-gap renormalization. Good qualitative agreement with experiment can be achieved in this way (Supplementary Figure 12A), although there is a mismatch in the crossing point of parallel and perpendicular polarizations. This crossing cannot be replicated by differences in the homogeneous and inhomogeneous broadening, reduced effective mass, or asymmetry in the effective masses of electrons and holes between the two experiments. A more quantitative fit can however be achieved if the effective carrier temperatures can differ for different polarizations (Supplementary Figure 12B). This recreates the experimental data more effectively, and highlights that there is different spectral broadening in the parallel and perpendicular spectra. This leads to the intriguing possibility that the parallel and perpendicular carrier populations interact only weakly, and maintain different temperatures, or carrier-phonon interactions, for several picoseconds. The origin of such an effect is beyond the scope of this investigation, but may well be justified given that carrier-phonon scattering rates have been shown to be dependent on carrier density and that diffusion effects between nanoregions are expected to dominate only at time scales longer than the cooling times. ${ }^{5}$

\section{Discussion}

We report an optically excited picosecond transient anisotropy in the electronic states of lead halide perovskites. The picosecond timescale is orders of magnitude slower than the optical orientation observed in crystalline inorganic semiconductors ${ }^{18}$. Our results show that the anisotropy does not arise from the organic parts of the metal-halide perovskites. In agreement with modeling and first principles calculations, we conclude that the observed effects arise from dynamic structural anisotropies in the material that are sufficient to perturb the local band structure and accessible electronic states. This suggests that there is strong coupling between local anharmonic lattice dynamics ${ }^{9}$ and the optical properties of the electronic states in these materials, in contrast to classical crystalline semiconductors in which delocalized electronic states are rather insensitive to local lattice dynamics. Previous experiments and calculations suggest that dynamic deformations of small regions of the lead iodide lattice spontaneously lead to localized polarized distortions on picosecond timescales, with characteristic size of less than $10 \mathrm{~nm}^{9,41}$. Our work supports this interpretation, and we conclude that these distortions lead to carrier populations that interact only weakly over the timescales of lattice reorganization. We therefore consider each lead halide perovskite sample and crystallite to consist of multiple nanoregions, each with optical and electronic properties that dynamically fluctuate on picosecond timescales $8,15,42$ to create a dynamic landscape of electronic states. We suggest that these electronically distinct regions represent the fundamental units of perovskite electronic structure, rather than crystal domains or individual nanocrystals. Stabilizing the perovskite structure is therefore likely to increase the polarization anisotropy magnitude and lifetime. A possible mechanism is the application of suitable external strain onto the crystal lattice, for example through piezoelectric force. The soft nature of both hybrid and purely inorganic perovskites gives rise to behavior not observed in either classic organic or inorganic semiconductors, which has farreaching implications for the understanding and application of this important class of materials.

\section{Methods}

Steady state linear absorption. Steady state linear absorption measurements were performed using a custom-modified Zeiss Axio A1 optical microscope equipped with a digital CCD camera (IDS UI-3580LE). Each sample was illuminated in transmission from a halogen lamp (Zeiss HAL100). A linear polarizer (Zeiss A1 polarizer D 427706) in the illumination beam path defined the orientation of the incident polarization. The incident light was focused through a condenser onto the sample (Zeiss 424225-9001). The transmitted light was coupled into a $\times 05$, NA 0.13 objective (Zeiss EC Epiplan-Neofluar 1156-514). The reflected signal from the sample was filtered by a broadband wire grid linear polarizer (Thorlabs WP25M$\mathrm{UB})$. The polarizer was mounted on a motorized rotation stage on the optical path and rotated between the orientation parallel to the incident beam polarizer, and perpendicular to it. The central area of $\varnothing 300 \mu \mathrm{m}$ reflected from the illuminated area was coupled into a $600 \mu \mathrm{m}$ core optical fiber (Avantes FC-UV600) mounted in confocal configuration and measured in a spectrometer (Avantes Avaspec

HS2048). The sample was manually rotated by $90^{\circ}$ to check for evidence of static Kerr rotation.

Linear polarization sensitive transient absorption. The output of a Ti:Sapphire amplifier system (Spectra-Physics Solstice) operating at $1 \mathrm{kHz}$ and generating $90-\mathrm{fs}$ pulses was split into the pump and probe beam paths. The visible broadband probe beams were generated in home-built noncollinear optical parametric amplifiers. The visible narrowband ( $25 \mathrm{meV}$ full-width at half-maximum) pump beam was provided by a TOPAS optical parametric amplifier (Light Conversion). The transmitted pulses were collected with an InGaAs dual-line array detector (Hamamatsu G11608-512) driven and read out by a custom-built board from Stresing Entwicklungsbüro. The linear polarization of the pump beam was set at $45^{\circ}$ with respect to the probe beam before the sample. During the measurement none of the polarizers before the sample were varied. Polarization dependent measurements were carried out according to Tan et al. ${ }^{33}$ by rotating a linear polarizer located in the probe beam immediately after the sample. This polarizer was set to be either parallel or perpendicular to the pump polarization. A final linear polarizer fixed at $45^{\circ}$ to the variable polarizer was placed directly in front of the detector, in order to remove the effect of any detector polarization dependence. The chirp in all measurements were corrected by reference measurements of the coherent artifact on glass substrates. The chirp was corrected identically in the parallel and perpendicular measurements.

Data availability. All relevant data is available on the Cambridge Repository at the following link: https://doi.org/10.17863/CAM.26301.

Received: 17 November 2017 Accepted: 8 August 2018

Published online: 30 August 2018

\section{References}

1. Cho, H. et al. Overcoming the electroluminescence efficiency limitations of perovskite light-emitting diodes. Science 350, 1222-1225 (2015).

2. Lee, M. M., Teuscher, J., Miyasaka, T., Murakami, T. N. \& Snaith, H. J. Efficient hybrid solar cells based on meso-superstructured organometal halide perovskites. Science 338, 643-647 (2012).

3. Wehrenfennig, C., Eperon, G. E., Johnston, M. B., Snaith, H. J. \& Herz, L. M. High charge carrier mobilities and lifetimes in organolead trihalide perovskites. Adv. Mater. 26, 1584-1589 (2014).

4. Johnston, M. B. \& Herz, L. M. Hybrid perovskites for photovoltaics: chargecarrier recombination, diffusion, and radiative efficiencies. Acc. Chem. Res. 49, 146-154 (2016).

5. Price, M. B. et al. Hot-carrier cooling and photoinduced refractive index changes in organic-inorganic lead halide perovskites. Nat. Commun. 6, 8420 (2015).

6. Richter, J. M. et al. Enhancing photoluminescence yields in lead halide perovskites by photon recycling and light out-coupling. Nat. Commun. 7, 13941 (2016).

7. Guo, P. et al. Polar fluctuations in metal halide perovskites uncovered by acoustic phonon anomalies. ACS Energy Lett. 2, 2463-2469 (2017).

8. $\mathrm{Wu}, \mathrm{X}$. et al. Light-induced picosecond rotational disordering of the inorganic sublattice in hybrid perovskites. Sci. Adv. 3, 1-8 (2017).

9. Yaffe, O. et al. Local polar fluctuations in lead halide perovskite crystals. Phys. Rev. Lett. 118, 136001 (2017)

10. Miyata, K. et al. Large polarons in lead halide perovskites. Sci. Adv. 3, e1701217 (2017).

11. Zhu, H. et al. Screening in crystalline liquids protects energetic carriers in hybrid perovskites. Science 353, 1409-1413 (2016)

12. Even, J., Pedesseau, L., Jancu, J.-M. M. \& Katan, C. Importance of spin-orbit coupling in hybrid organic/inorganic perovskites for photovoltaic applications. J. Phys. Chem. Lett. 4, 2999-3005 (2013). 
13. Kim, M., Im, J., Freeman, A. J., Ihm, J. \& Jin, H. Switchable $S=1 / 2$ and $J=1 / 2$ Rashba bands in ferroelectric halide perovskites. Proc. Natl Acad. Sci. USA 111, 6900-6904 (2014)

14. Kepenekian, M. \& Even, J. Rashba and Dresselhaus couplings in Halide perovskites: accomplishments and opportunities for spintronics and spin-orbitronics. J. Phys. Chem. Lett. 8, 3362-3370 (2017).

15. Zheng, F., Tan, L. Z., Liu, S. \& Rappe, A. M. Rashba spin-orbit coupling enhanced carrier lifetime in $\mathrm{CH}_{3} \mathrm{NH}_{3} \mathrm{PbI}_{3}$. Nano Lett. 15, 7794-7800 (2015).

16. Isarov, M. et al. Rashba effect in a single colloidal $\mathrm{Cs} \mathrm{PbBr}_{3}$ perovskite nanocrystal detected by magneto-optical measurements. Nano Lett. 17, 5020-5026 (2017).

17. Niesner, D. et al. Giant Rashba splitting in $\mathrm{CH}_{3} \mathrm{NH}_{3} \mathrm{PbBr}_{3}$ organic-inorganic perovskite. Phys. Rev. Lett. 117, 1-6 (2016).

18. Meier, F. \& Zakharchenya, B. Optical Orientation (North Holland, 1984).

19. Zemskii, V., Zakharchenya, B. \& Mirlin, D. Polarization of hot photoluminescence in semiconductors of the GaAs type. JETP Lett. 24, 82 (1976).

20. Oudar, J. L. et al. Femtosecond orientational relaxation of photoexcited carriers in GaAs. Phys. Rev. Lett. 53, 384-387 (1984).

21. Portella, M. T., Bigot, J.-Y., Schoenlein, R. W., Cunningham, J. E. \& Shank, C. V. k-space carrier dynamics in GaAs. Appl. Phys. Lett. 60, 2123 (1992).

22. Ramsdale, C. M. \& Greenham, N. C. Ellipsometric determination of anisotropic optical constants in electroluminescent conjugated polymers. Adv. Mater. 14, 212-215 (2002)

23. Du, K. Z., Meng, W., Wang, X., Yan, Y. \& Mitzi, D. B. Bandgap engineering of lead-free double perovskite $\mathrm{Cs}_{2} \mathrm{AgBiBr}_{6}$ through trivalent metal alloying. Angew. Chem. 56, 8158-8162 (2017).

24. Brau, A., Bruesch, P., Farges, J. P., Hinz, W. \& Kuse, D. Polarized optical reflection spectra of the highly anisotropic organic semiconductor TEA (TCNQ)2. Phys. Status Solidi 62, 615-623 (1974).

25. Minemawari, H. et al. Inkjet printing of single-crystal films. Nature 475, 364-367 (2011).

26. Eperon, G. E., Burlakov, V. M., Docampo, P., Goriely, A. \& Snaith, H. J. Morphological control for high performance, solution-processed planar heterojunction perovskite solar cells. Adv. Funct. Mater. 24, 151-157 (2014).

27. Protesescu, L. et al. Nanocrystals of cesium lead halide perovskites (CsPbX 3, $\mathrm{X}=\mathrm{Cl}, \mathrm{Br}$, and $\mathrm{I}$ ): novel optoelectronic materials showing bright emission with wide color gamut. Nano Lett. 15, 3692-3696 (2015).

28. Bae, W. K. et al. R/G/B/natural white light thin colloidal Quantum dot-based light-emitting devices. Adv. Mater. 26, 6387-6393 (2014).

29. Jasieniak, J., Califano, M. \& Watkins, S. E. Size-dependent valence and conduction band-edge energies of semiconductor nanocrystals. ACS Nano 5, 5888-5902 (2011).

30. Yang, Y. et al. Observation of a hot-phonon bottleneck in lead-iodide perovskites. Nat. Photonics 10, 53-59 (2016).

31. Rivett, J. P. H., Richter, J. M., Price, M. B., Credgington, D. \& Deschler, F. Carrier-phonon interactions in hybrid halide perovskites probed with ultrafast anisotropy studies. In Physical Chemistry of Interfaces and Nanomaterials XV Vol. 9923 (eds Bakulin, A. A., Lovrincic, R. \& Banerii, N.) (SPIE, 2016).

32. Sheng, C. et al. Exciton versus free carrier photogeneration in organometal trihalide perovskites probed by broadband ultrafast polarization memory dynamics. Phys. Rev. Lett. 114, 116601 (2015).

33. Tan, H.-S., Piletic, I. R. \& Fayer, M. D. Polarization selective spectroscopy experiments: methodology and pitfalls. J. Opt. Soc. Am. B 22, 2009 (2005).

34. Mattoni, A., Filippetti, A., Saba, M. I. \& Delugas, P. Methylammonium rotational dynamics in lead halide perovskite by classical molecular dynamics: the role of temperature. J. Phys. Chem. C 119, 17421-17428 (2015).

35. Kawai, H., Giorgi, G., Marini, A. \& Yamashita, K. The mechanism of slow hothole cooling in lead-iodide perovskite: first-principles calculation on carrier lifetime from Electron-Phonon interaction. Nano Lett. 15, 3103-3108 (2015).

36. Filippetti, A., Mattoni, A., Caddeo, C., Saba, M. I. \& Delugas, P. Low electronpolar optical phonon scattering as a fundamental aspect of carrier mobility in methylammonium lead halide $\mathrm{CH}_{3} \mathrm{NH}_{3} \mathrm{PbI}_{3}$ perovskites. Phys. Chem. Chem. Phys. 18, 15352-15362 (2016).

37. Li, M. et al. Slow cooling and highly efficient extraction of hot carriers in colloidal perovskite nanocrystals. Nat. Commun. 8, 3-11 (2017).
38. Selig, O. et al. Organic cation rotation and immobilization in pure and mixed methylammonium lead-halide perovskites. J. Am. Chem. Soc. 139, 4068-4074 (2017).

39. Quarti, C., Mosconi, E. \& De Angelis, F. Structural and electronic properties of organo- halide hybrid perovskites from ab initio molecular dynamics. Phys. Chem. Chem. Phys. 17, 9394-9409 (2015).

40. Richter, J. M. et al. Ultrafast carrier thermalization in lead iodide perovskite probed with two-dimensional electronic spectroscopy. Nat. Commun. 8, 376 (2017).

41. Beecher, A. N. et al. Direct observation of dynamic symmetry breaking above room temperature in methylammonium lead iodide perovskite. ACS Energy Lett. 1, 880-887 (2016).

42. Etienne, T., Mosconi, E. \& De Angelis, F. Dynamical origin of the Rashba effect in organohalide lead perovskites: a key to suppressed carrier recombination in perovskite solar cells? J. Phys. Chem. Lett. 7, 1638-1645 (2016).

\section{Acknowledgements}

We thank Richard T. Phillips, Richard H. Friend, Johannes M. Richter, Kai Chen and Justin M. Hodgkiss for helpful discussions and advice, and Silvia Vignolini for lab access J.P.H.R., R.M. and J.X. acknowledge support from the EPSRC Cambridge NanoDTC, under grant EP/G037221/1 and EP/L015978/1. L.T. and A.R. acknowledge support from the Office of Naval Research, under grant N00014-17-1-2574. M.B.P. acknowledges support from the EPSRC. N.J.L.K.D. acknowledges funding from the Ernest Oppenheimer fund. D.C. acknowledges support from the Royal Society (grant nos. UF130278 and RG140472). F.D. acknowledges funding from a Herchel Smith Research Fellowship and an Advanced Research Fellowship from the Winton Programme for the Physics of Sustainability.

\section{Author contributions}

J.P.H.R., F.D., and S.B. performed polarization selective transient absorption measurements. L.T. and A.R. performed the DFT calculations. M.B.P. performed the modeling. R. M. performed the steady state linear absorption measurements. J.P.H.R., N.J.L.K.D., J.X., and Y.Z. prepared the samples. A.R., B.S., D.C., and F.D. supervised the work. All authors discussed the data and contributed to the manuscript.

\section{Additional information}

Supplementary Information accompanies this paper at https://doi.org/10.1038/s41467 018-06009-3.

Competing interests: The authors declare no competing interests.

Reprints and permission information is available online at http://npg.nature.com/ reprintsandpermissions/

Publisher's note: Springer Nature remains neutral with regard to jurisdictional claims in published maps and institutional affiliations.

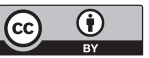

Open Access This article is licensed under a Creative Commons Attribution 4.0 International License, which permits use, sharing, adaptation, distribution and reproduction in any medium or format, as long as you give appropriate credit to the original author(s) and the source, provide a link to the Creative Commons license, and indicate if changes were made. The images or other third party material in this article are included in the article's Creative Commons license, unless indicated otherwise in a credit line to the material. If material is not included in the article's Creative Commons license and your intended use is not permitted by statutory regulation or exceeds the permitted use, you will need to obtain permission directly from the copyright holder. To view a copy of this license, visit http://creativecommons.org/ licenses/by/4.0/.

(C) The Author(s) 2018 